\title{
Perfil epidemiológico dos casos notificados da esquistossomose mansoni ocorridos no
}

\section{Brasil}

\author{
Epidemiological profile of notified cases of schistosomiasis mansoni occurring in Brazil \\ Perfil epidemiológico de casos notificados de cistosomiasis mansoni que ocurren en Brasil
}

Recebido: 13/11/2021 | Revisado: 17/11/2021 | Aceito: 20/11/2021 | Publicado: 02/12/2021

Martiniano de Araújo Rocha ORCID: https://orcid.org/0000-0003-4419-8672 Universidade Ceuma, Brasil

E-mail: martinianoaraujo8@gmail.com

Lorena Lima Gouveia de Oliveira ORCID: https://orcid.org/0000-0003-1124-8629 Universidade Ceuma, Brasil

E-mail: lorena115249@ ceuma.com.br

Maria Fernanda Ribeiro Rocha ORCID: https://orcid.org/0000-0001-6699-5416 Universidade Ceuma, Brasil

E-mail: mariafnandarr@bol.com.br

Emilly Rafaela Rodrigues Jorge ORCID: https://orcid.org/0000-0002-5157-5483 Universidade Ceuma, Brasil

E-mail: eemillyrafaela@gmail.com

Hioara Kely Arcanjo da Silva ORCID: https://orcid.org/0000-0002-3249-1487 Universidade Ceuma, Brasil

E-mail: hioarasfm@hotmail.com Andressa Santos Silva

ORCID: https://orcid.org/0000-0002-3158-3670 Universidade Ceuma, Brasil E-mail: andressasnts0@gmail.com

Maria Clara Duarte de Melo ORCID: https://orcid.org/0000-0003-2277-1925 Universidade Ceuma, Brasil

E-mail: mariaclara117206@ceuma.com.br Marta Gomes Rocha

ORCID: https://orcid.org/0000-0002-6375-7727 Universidade Ceuma, Brasil

E-mail: martarocha.mgr@gmail.com

Eva Maria Oliveira Cutrim Dantas ORCID: https://orcid.org/0000-0001-6720-1331 Universidade Ceuma, Brasil

E-mail: evacutrim@hotmail.com

Marluce Leite De Alencar ORCID: https://orcid.org/0000-0002-6964-6016 Universidade Ceuma, Brasil

E-mail: enfermeiratrabalho23@gmail.com

Jordana Santos Sousa Silva ORCID: https://orcid.org/0000-0001-6645-0192 Universidade Ceuma, Brasil

E-mail: jordanna21@gmail.com

Rebeca Aranha Araújo

ORCID: https://orcid.org/0000-0002-9668-7550 Universidade Ceuma, Brasil

E-mail: rebeca117289@ceuma.com.br

Gabriella Santana Aragão ORCID: https://orcid.org/0000-0002-9529-3568 Universidade Ceuma, Brasil

E-mail: gabriella117809@ceuma.com.br

Josianny Liérgine Vasconcelos Fernandes Freitas ORCID: https://orcid.org/0000-0003-3585-7998

Universidade Ceuma, Brasil

E-mail: josianny.fernandes@hotmail.com 
Ermilton Junio Pereira de Freitas ORCID: https://orcid.org/0000-0001-8391-1026 Universidade Ceuma, Brasil

E-mail: ermilton.freitas@ ceuma.br

\begin{abstract}
Resumo
Objetivo: descrever o perfil epidemiológico dos casos notificados da esquistossomose mansoni ocorridos no Brasil no período compreendido entre 2013 e 2017. Métodos: Caracteriza-se como um estudo descritivo e retrospectivo, desenvolvido a partir de dados secundários coletados no SINAN. Os dados foram obtidos no software Microsoft Excel®. A pesquisa não exigiu avaliação por Comitê de Ética em Pesquisa. Resultados: Os dados de incidência no período do estudo acumularam 28.781 casos. A região Sudeste se destacou com o maior número de casos, seguida pelas regiões Nordeste, Norte, Centro-Oeste e Sul. Minas Gerais, São Paulo e Bahia foram os que apresentaram maior incidência da doença, enquanto os estados do Acre, Roraima e Pará apresentaram as menores taxas. Dentre as variáveis avaliadas, em associação à doença parasitária, houve maior predominância do sexo masculino, adultos, cor parda e com baixa escolaridade. Considerações finais: o Brasil, apesar de ter apresentado tendência decrescente no número de casos nos últimos anos, o número de casos ainda é considerado alto e, portanto, necessita estabelecer melhores políticas de saúde voltadas à promoção, prevenção e controle da esquistossomose.
\end{abstract}

Palavras-chave: Doença negligenciada; Epidemiologia; Esquistossomose mansoni.

\begin{abstract}
Objective: to describe the epidemiological profile of reported cases of schistosomiasis mansoni that occurred in Brazil in the period between 2013 and 2017. Methods: It is characterized as a descriptive and retrospective study, developed from data collected in SINAN. Data were obtained using Microsoft Excel@ software. The research did not require evaluation by the Research Ethics Committee. Results: Incidence data in the study period accumulated a total of 28,781 cases. The Southeast region stood out with the highest number of cases, followed by the Northeast, North, Midwest and South regions. Minas Gerais, São Paulo and Bahia were the ones with the highest incidence of the illness, while the states of Acre, Roraima and Pará had the lowest rates. Among the variables evaluated, in association with parasitic disease, there was a greater predominance of males, adults, brown color and with low education. Final considerations: Brazil, despite having shown a decreasing trend in the number of cases in recent years, the number of cases is still considered high and, therefore, needs to establish better health policies aimed at the promotion, prevention and control of schistosomiasis.
\end{abstract}

Keywords: Neglected disease; Epidemiology; Schistosomiasis mansoni.

\begin{abstract}
Resumen
Objetivo: describir el perfil epidemiológico de los casos notificados de esquistosomiasis mansoni ocurridos en Brasil en el período entre 2013 y 2017. Métodos: Se caracteriza por ser un estudio descriptivo y retrospectivo, desarrollado a partir de datos recolectados en el SINAN. Los datos se obtuvieron mediante el software Microsoft Excel®. La investigación no requirió evaluación por parte del Comité de Ética en Investigación. Resultados: Los datos de incidencia durante el período de estudio acumularon 28.781 casos. La región Sudeste se destacó con el mayor número de casos, seguida de las regiones Nordeste, Norte, Medio Oeste y Sur. Minas Gerais, São Paulo y Bahía fueron las de mayor incidencia de enfermedad, mientras que los estados de Acre, Roraima y Pará tenía las tasas más bajas. Entre las variables evaluadas, en asociación con enfermedad parasitaria, hubo mayor predominio de varones, adultos, de color marrón y con bajo nivel educativo. Consideraciones finales: Brasil, a pesar de haber mostrado una tendencia decreciente en el número de casos en los últimos años, el número de casos aún se considera alto y, por lo tanto, necesita establecer mejores políticas de salud dirigidas a la promoción, prevención y control de la esquistosomiasis.
\end{abstract}

Palabras clave: Enfermedad desatendida; Epidemiología; Esquistosomiasis mansoni.

\title{
1. Introdução
}

A esquistossomose é uma das doenças parasitárias que mais afetam o homem, ocupando o segundo lugar, acometendo cerca de 240 milhões de indivíduos a nível global, sendo antecedida apenas pela malária, em relação a magnitude e repercussão como uma das principais doenças infecciosas do mundo (LoVerde, 2019; Soares, et al., 2019). A nível nacional, o Brasil apresentou nas regiões nordeste e sudeste entre 2013-2017, um total de 6399 e 21.464 casos respectivamente (Sobrinho, et al., 2020; Costa \& Filho, 2021). É uma doença tropical, causada por vermes parasitas com ampla distribuição geográfica atingindo partes do Oriente Médio, América do Sul, Sudeste Asiático e, particularmente, a África Subsaariana (Mcmanus, et al., 2018). O Brasil possui uma diversidade de caramujos de límnicos do gênero Biomphalaria, sendo os três hospedeiros intermediários neotropicais o B. glabrata, B. straminea e B. tenagophila (Palasio, et al., 2017). 
Caracterizada como doença negligenciada, a esquistossomose, ocorre em quase todo o território brasileiro, possuindo natureza parasitária e veiculação hídrica, sendo causada pelo trematódeo digenético Schistosoma mansoni (GOMES, et al., 2016). É pertencente à família Schistosomatidae, apresentando como característica a existência de sexos separados, com nítido dimorfismo sexual. Este parasita possui o ser humano como hospedeiro definitivo, onde realiza a maturação e reprodução sexuada, e os caramujos do gênero Biomphalaria como hospedeiros intermediários, no qual aloja o parasita em sua forma larval e desenvolve a proliferação assexuada (Souza, et al., 2017).

O ciclo da enfermidade inicia quando o ser humano entra em contato com cercárias, que penetram na pele. Em seguida, as mesmas se diferenciam em larvas denominadas esquistossômulos, que atingem o sangue, e pelas veias, são transportadas para a circulação arterial em direção ao fígado. Nesse local ocorre o amadurecimento em vermes adultos e na sequência seu alojamento nas vênulas mesentéricas, onde a fêmea libera seus ovos fertilizados, que penetram no endotélio vascular e atingem o lúmen do intestino ou a bexiga. Na sequência, os ovos são excretados nas fezes ou na urina, e devem alcançar a água doce para poderem eclodir. Uma vez rompidos, os miracídios (larvas ciliadas) penetram nos caramujos, onde se desenvolvem e multiplicam-se produzindo novas cercárias, que uma vez liberadas podem penetrar na pele humana dando continuidade ao ciclo da doença (Leivison, 2016; Nelwan, 2019).

Os achados patológicos da enfermidade referem-se a presença de ovos no fígado, no baço ou na parede do intestino ou da bexiga. Os ovos induzem os granulomas, fibrose, hepatomegalia e esplenomegalia. Em relação aos achados clínicos, a maioria dos pacientes é assintomática, porém infecções crônicas podem tornar-se sintomáticas. Após a penetração das cercárias, os sintomas consistem no prurido e dermatite. Nas duas ou três semanas seguintes, febre, calafrios, diarreia, linfadenopatia e hepatoesplenomegalia podem ser desenvolvidos. Além disso, em estágios crônicos da doença a morbidade e mortalidade podem ser significativas (Leivison, 2016). Todavia, apesar da magnitude dos efeitos da doença, o tratamento é realizado pelo uso do praziquantel, uma droga de baixo custo e distribuída gratuitamente pelo Sistema Único de Saúde (Silva, Neto \& Silva, 2021).

No Brasil, a persistência da esquistossomose está condicionada a um conjunto de dimensões que transcendem o fenômeno biológico. A pobreza, ambientes adversos, falta de educação e conscientização, continuam sendo problemas para o controle e erradicação da esquistossomose. Dentre os fatores que favorecem sua instalação destacam-se a utilização de águas contaminadas para o exercício da agricultura, trabalho doméstico e lazer são fatores importantes a serem considerados. Somando-se a isso, dificuldades de acesso aos serviços de saúde, favorecem a disseminação da doença e a progressão do número de casos, e tem levado o Brasil à marca do país com maior número de casos nas Américas (Verjee, 2019; Rocha, et al., 2016).

Dessa forma, considerando essa problemática, o presente estudo tem por objetivo descrever o perfil epidemiológico dos casos notificados da esquistossomose mansoni ocorridos no Brasil no período compreendido entre 2013 e 2017.

\section{Metodologia}

O presente estudo caracterizou-se como uma pesquisa descritiva e retrospectiva, que visou verificar a dinâmica dos casos de esquistossomose no Brasil, entre os anos de 2013 e 2017. O Brasil possui área territorial de $8.515 .767,049 \mathrm{~km}^{2}$, sendo a estimativa da população de 211.755.692 milhões de habitantes. O país, devido a vasta abrangência territorial é subdividido em cinco grandes regiões: Norte, Nordeste, Sul, Sudeste e Centro-Oeste. A divisão administrativa consiste em 26 estados e 1 Distrito Federal, acumulando um total de 5.570 municípios por todo o território.

O estudo foi desenvolvido a partir de dados secundários, colhidos no Sistema de Informação de Agravos de Notificação - SINAN ((http://portalsinan.saude.gov.br/dados-epidemiologicos-sinan). Esse sistema, do Ministério da saúde, é alimentado, principalmente, pela notificação e investigação de casos de doenças e agravos que constam da lista nacional de 
doenças de notificação compulsória.

Desse banco de dados foram utilizadas informações referentes aos casos confirmados por estados e regiões do Brasil, como também informações relacionadas ao sexo, faixa etária, cor e escolaridade dos pacientes acometidos. Para a análise da variável idade, foram estabelecidas classes de acordo com as seguintes classes etárias: jovens $(0<\mathrm{X} \leq 14)$, adultos $(15 \leq \mathrm{X} \leq$ $59)$ e idosos $(60 \leq X)$. Acerca da variável cor/etnia realizou-se a distribuição dos casos positivos em cinco grandes grupos, sendo eles, branca; preta; amarela; parda; e indígena. Já em relação a variável escolaridade foram dispostas as seguintes divisões: Classe I (analfabetos e ensino fundamental incompleto); Classe II (ensino fundamental completo e ensino médio incompleto); Classe III (ensino médio completo, ensino superior completo e incompleto).

Os dados obtidos foram tabulados em gráficos e tabelas, e foram construídos para compilar as informações utilizando o software Microsoft Excel ${ }^{\circledR}$, como desenvolvido por estudos semelhantes realizados por Araújo et. al (2019), Sobrinho, et al. (2020) e Costa e Filho (2021). Em detrimento do presente estudo utilizar apenas dados secundários, não foi necessária a submissão para apreciação pelo Comitê de Ética em Pesquisa (CEP).

Ademais, os estudos utilizados para a discussão dos resultados foram alcançados por meio das plataformas Google Scholar, Scientific Electronic Library Online (SCIELO), PubMed e Biblioteca Virtual em Saúde (BVS). Os critérios de inclusão foram artigos publicados nos últimos cinco anos de referência (2016-2021), disponibilizados de forma gratuita e escritos em português, inglês ou espanhol. Já os artigos excluídos foram aqueles que não abordavam a temática do estudo, e que não contribuíam para a discussão do trabalho.

\section{Resultados}

A análise dos dados de ocorrência revelou um total 28.781 (média $=5.756,2 \pm 960,04$ casos), com destaque para os 3 primeiros anos do período de estudo 2013, 2014 e 2015, que contribuíram com 6.434 (22,36\%), 6.466 (22,47\%) e 6.382 $(22,17 \%)$ dos casos positivos. Já os dois anos subsequentes, 2016 e 2017, acumularam, respectivamente, 5.140 e 4.359 casos da doença (Figura 1).

Figura 1: Incidência dos casos de esquistossomose no Brasil entre os anos de 2013 e 2017.

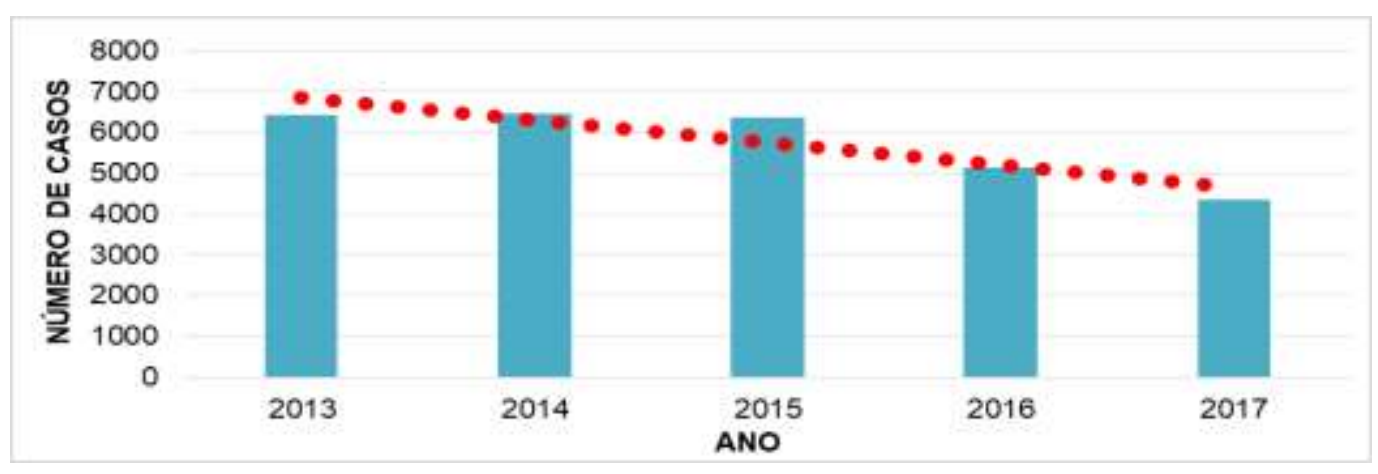

Fonte: Autores (2021).

A análise dos casos por regiões do Brasil, mostrou diferenças importantes na ocorrência entre elas (Figura 2). A região sudeste se destacou disparadamente com o maior número de casos $(\mathrm{n}=21.464,74,58 \%)$, seguida da região nordeste ( $\mathrm{n}$ $=6.399,22,23 \%)$ e região norte $(n=335,1,16 \%)$. As regiões centro oeste e sul juntas acumularam cerca de $2 \%$ dos casos totais ( $n=331$ e 252 , respectivamente).

Com relação ao número de casos por estado, a análise revelou importante centros com maior frequência, (Figura 3) dos quais, Minas Gerais, São Paulo e Bahia, foram os que apresentaram maior incidência da enfermidade, com os seguintes 
valores, respectivos 15.724 (Média = 3.144,8); 3.333 (Média = 666,6) e, 3.226 (Média = 645,2). Já os estados Acre com nenhum caso, Roraima e Pará com apenas um caso cada foram os que apresentaram menor incidência.

Figura 2. Número de casos de esquistossomose no Brasil entre os anos de 2013 e 2017 registrados por região.

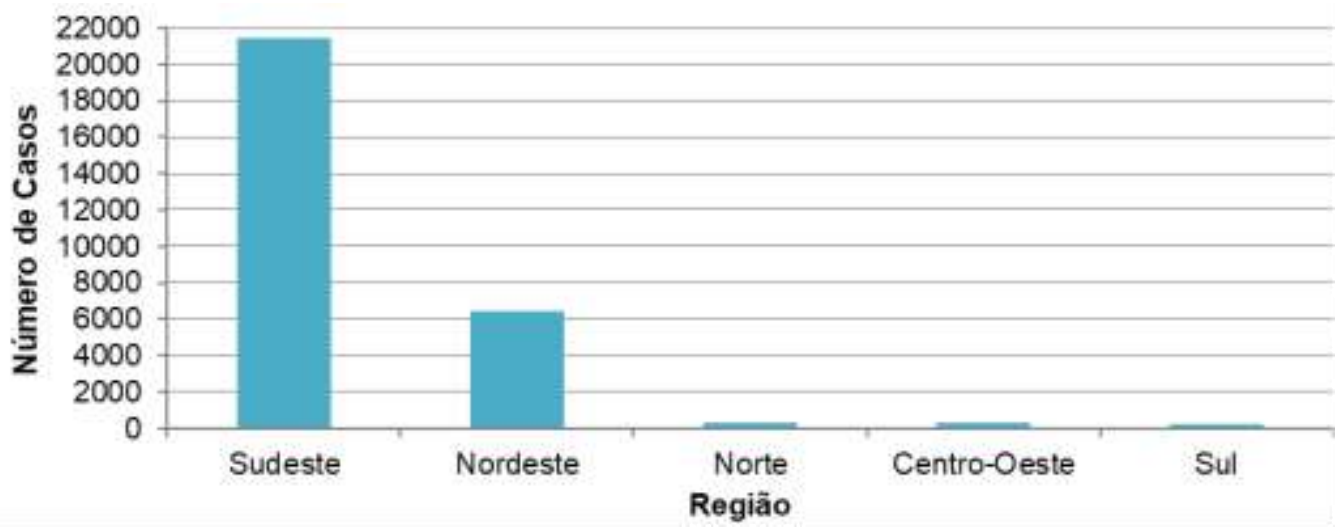

Fonte: Autores (2021).

Figura 3. Número de casos de esquistossomose no Brasil entre os anos de 2013 e 2017 registrados por estado da federação.

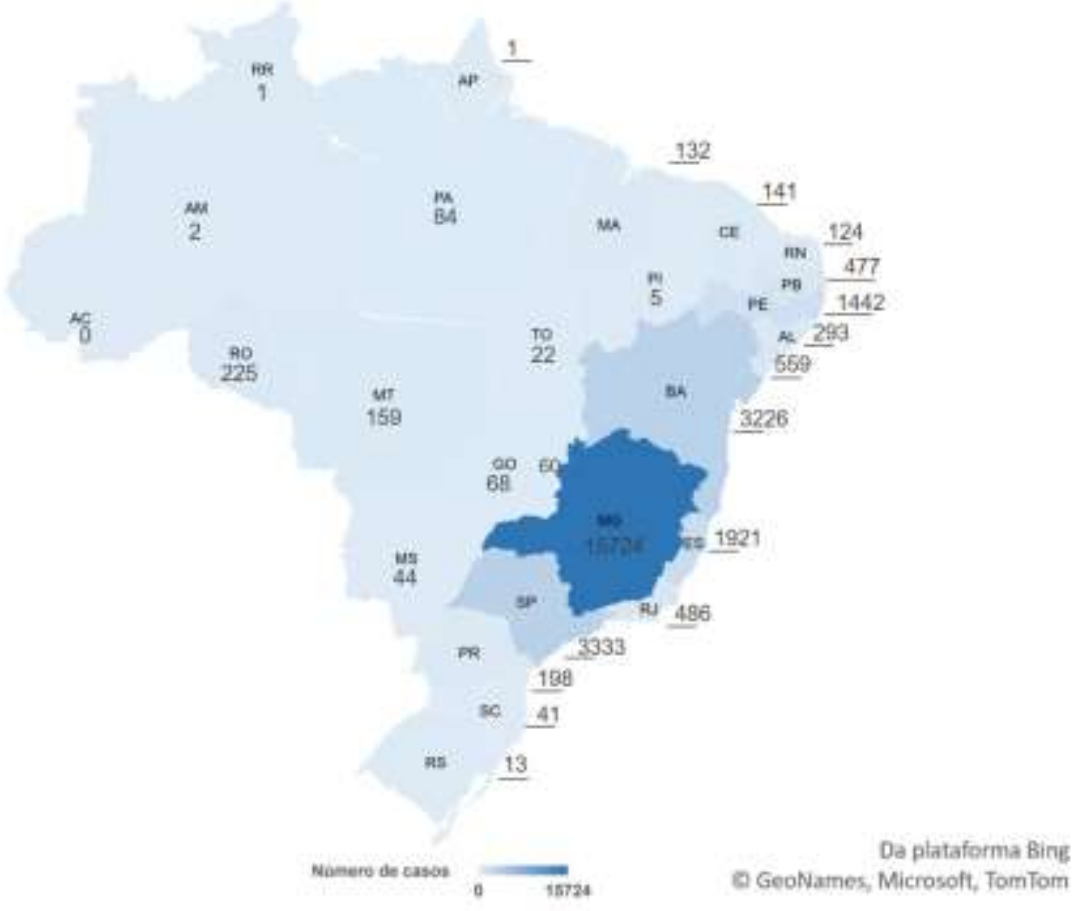

Fonte: Autores (2021).

$\mathrm{Na}$ análise por gênero, o Brasil revelou que os homens têm sido mais acometidos que as mulheres com 17.726 $(61,59 \%)$ e $11.053(38,40 \%)$ casos respectivamente (Tabela 1). Na análise desse parâmetro por região o padrão permaneceu, apresentando valores análogos ao perfil geral. Vale observar que no período analisado, apenas em dois casos não foram obtidos o sexo do paciente, todavia, mediante a essa parcela razoavelmente inexpressiva, não houve prejuízos consideráveis no resultado. 
Tabela 1 - Caracterização dos casos de esquistossomose, n=28.781. Brasil, 2013-2017

\begin{tabular}{lll}
\hline Variável & $\mathbf{N}$ & \% \\
\hline Sexo & & \\
Masculino & 17.726 & 61,59 \\
Feminino & 11.053 & 38,40 \\
Ignorados & 2 & 00,01 \\
\hline Idade & & \\
Jovens 00 - 14 & 3098 & 10,76 \\
Adultos 15 -59 & 22027 & 76,53 \\
Idosos 60 - 100 & 3655 & 12,70 \\
Ignorados & 1 & 00,00 \\
\hline Cor & & \\
Branca & 8121 & 28,22 \\
Preta & 2545 & 08,84 \\
Amarela & 402 & 01,40 \\
Pardo & 13685 & 47,55 \\
Indígena & 93 & 00,32 \\
Ignorados & 3935 & 13,67 \\
Escolaridade & & \\
CLASSE I & 9703 & 33,71 \\
CLASSE II & 3204 & 11,13 \\
CLASSE III & 3718 & 12,92 \\
Ignorados & 12156 & 42,24 \\
\hline
\end{tabular}

Fonte: Autores (2021).

Com relação à distribuição de casos por idade no país (Tabela 1), foi possível verificar que a maior incidência ocorreu em adultos $(n=22.027,76,54 \%)$, seguidos de idosos $(n=3.655,12,70 \%)$ e jovens $(n=3.098,10,76 \%)$. Na análise dessa variável por região, houve a mesma tendência, apresentando valores semelhantes ao perfil geral, com destaque apenas para a região sudeste, onde os o número de casos entre jovens ultrapassou a quantidade de casos em idosos. É importante destacar que no período analisado, apenas um caso não foi informado o valor da idade do paciente, contudo, mediante a essa parcela razoavelmente inexpressiva, não houve prejuízos consideráveis no estudo do parâmetro.

Em relação a cor/etnia da população acometida pela esquistossomose mansônica (Tabela 1), foi possível observar a seguinte distribuição de forma crescente: indígena $(n=93 ; 0,32 \%)$; amarela $(n=402 ; 1,40 \%)$; preta $(n=2.545 ; 8,84 \%)$; branca $(\mathrm{n}=8.121 ; 28,22 \%)$; e parda $(\mathrm{n}=13.685 ; 47,55 \%)$. É relevante mencionar ainda, que no período analisado, um total de 3.935 casos $(13,67 \%)$ foram ignorados, em outras palavras, as informações sobre a cor/etnia não foram computadas. Assim, percebese que, em detrimento da parcela considerável de casos ignorados, existe uma lacuna no estudo acerca dessa variável, pois a mesma foi influenciada significantemente pela não notificação dos dados.

Em relação à distribuição de casos por grau de escolaridade no país (Tabela 1), foi possível verificar a maior incidência em indivíduos da classe $\mathrm{I}(\mathrm{n}=9.703 ; 58,36 \%)$, que compreende os indivíduos analfabetos e que possuem ensino fundamental incompleto; seguido da classe III $(n=3.718 ; 22,36 \%)$, que abrange os indivíduos que possuem ensino médio completo, ensino superior completo ou incompleto; e por fim a classe II ( $\mathrm{n}=3.204 ; 19,27 \%)$, que contemplam a população que possui ensino fundamental completo e ensino médio incompleto. Na análise por região o padrão permaneceu, apresentando valores similares ao retrato geral do país. Cabe ainda ponderar que um total de 12.156 casos não foi considerado para o cálculo da distribuição, devido a não notificação no sistema. 


\section{Discussão}

Com os dados obtidos foi observado que o Brasil, no período entre 2013-2017, apresentou uma tendência reducional no número de casos de esquistossomose mansoni, representada pela linha vermelha pontilhada (Figura 1). Tal fato pode estar correlacionado com a aprovação da resolução, realizada pela Organização Mundial da Saúde (OMS) na $66^{\circ}$ Assembleia Mundial da Saúde, que determinou o Plano de Ação para a Prevenção e Controle de Doenças Não Transmissíveis 2013 - 2020 que se concentra no controle dos determinantes sociais em saúde. Esse documento prevê a promoção do acesso às drogas essenciais para o tratamento do problema da esquistossomose, além da mobilização de recursos para sustentar as atividades de controle, saneamento básico e educação em saúde. Ademais, entre os anos de 2013 e 2018 ações interventivas como a “Campanha Nacional de Hanseníase, Verminoses, Tracoma e Esquistossomose "de fato contribuíram para a redução no número de casos, uma vez que objetivavam aumentar a identificação de casos em escolas da rede pública de ensino (Brasil, 2021).

Ainda sobre essa perspectiva, interpreta-se que o Brasil caminha para o controle da doença. Todavia, fazendo uma análise comparativa entre as regiões que fazem parte do país, a mesma tendência decrescente não foi evidenciada. As regiões que indicaram um aumento da incidência de casos em relação ao ano inicial analisado no estudo (2013) foram as regiões Nordeste, Norte, e Sul. Com destaque para a região norte, que apesar de ainda apresentar poucas ocorrências, no período compreendido, cresceu de forma alarmante de 42 para 100 casos. Tal evento pode estar ligado com o aumento da obrigatoriedade em realizar todas as notificações de forma completa no SINAN, bem como pelo avanço e praticidade dos métodos de diagnóstico e tratamento que são preconizados pelo Sistema Único de Saúde (Brasil, 2021).

Ademais, as regiões que apresentaram redução dos casos no período de referência foram as regiões centro-oeste e sudeste. Com destaque para a região sudeste, que é a região mais acometida, e que foi, em linhas gerais, a grande contribuinte para a redução dos casos no Brasil. No primeiro ano do estudo, a região sudeste apresentou incidência de 5098 casos e no último ano verificado (2017), foi constatado 2911 casos, corroborando a efetividade e sucesso das medidas de controle e prevenção da doença. Além disso, em um estudo semelhante desenvolvido por Costa e Filho (2021), também foi constato a diminuição da incidência dos casos.

A análise das progressões dos casos por estado, revelou uma tendência decrescente dos casos nas unidades federativas que mais contribuíam para o quadro geral dos casos. Com destaque para Bahia, São Paulo e Minas Gerais, que em 2013 apresentavam 695, 888 e 3.646 casos, e no ano de 2017 notificaram 536, 578 e 1.941, correspondendo a um percentual de redução dos casos significativo. Dessa maneira, apesar da redução, ainda é imprescindível a adoção das medidas de controle e intervenções guiadas pelo Ministério da Saúde, para evitar um novo surto da doença.

Em relação a variável gênero, foi verificado uma maior predominância da parasitose no sexo masculino, apresentando como percentual cerca de $61,59 \%$ dos casos. Outros estudos como os realizados por Santos et al. (2016) e Silva (2020) corroboram os resultados observados nesse trabalho. O maior acometimento masculino pode estar atrelado a dificuldade do acesso aos serviços de saúde e o paradigma relacionado a esse gênero. O modelo ainda patriarca observado na sociedade, onde o homem procurar por ações preventivas de saúde, ser interpretado como sinônimo de fraqueza ou sensibilidade é um dos fatores contribuintes para persistência da enfermidade, sobretudo nesse grupo (Gomes, et al., 2016). Desse modo, é inquestionável a urgência de traçar novas estratégias que visem abranger os homens enquanto potenciais usuários do Sistema Único de Saúde, para alcançar uma maior qualidade de vida e prevenir eventuais doenças.

A respeito do parâmetro idade, foram constatados ao longo dos cinco anos, uma maior frequência de casos na população pertencente a faixa etária adulta $(15 \leq \mathrm{X} \leq 59)$, correspondendo a um percentual equivalente a $76,54 \%$ dos casos. A mesma prevalência foi observada no estudo realizado na Bahia por Santos e Cardoso (2020). Tal condição pode ser explicada, pelo fato de ser uma população mais ativa e estarem mais expostos a enfermidade, como em profissões como a agricultura, a 
pecuária e a pesca, sendo por conseguinte, uma população mais suscetível a desenvolver a doença (Costa \& Filho, 2021).

Acerca da distribuição dos casos em relação a cor/etnia dos indivíduos acometidos pelo schistosoma, foi averiguado a persistência da doença principalmente na população parda e negra, ratificando estudos realizados por Junior et al. (2017) e Araújo et. al (2019). Contudo, a prevalência da esquistossomose nesses grupos transcende a esfera da coloração da pele, uma vez que no Brasil, o baixo nível socioeconômico e o reduzido grau de escolaridade, geralmente são atribuídos a essa parcela, ratificando ainda a persistência desse estigma social que coopera para que está população seja mais carente em parâmetros de condições de habitação, assistência em saúde, conhecimento sobre hábitos de higiene pessoal e de políticas públicas de saneamento básico, tratamento de esgoto e tratamento de água (Melo, et al., 2018; Barbosa \& Silva, 2018).

A distribuição dos casos pelo grau de escolaridade dos pacientes acometidos com esquistossomose revelou uma incidência maior conforme o nível de ensino fosse mais inferior. Em consonância com o estudo desenvolvido por Melo et al. (2018), e Carvalho e Siqueira (2019), o grau de instrução dos indivíduos em relação a doença é um fator imprescindível no controle da parasitose, pois o acesso à informação proporciona o empoderamento da população, um maior entendimento sobre a importância dos cuidados de saúde, bem como sobre comportamentos de higiene pessoal, que são medidas necessárias não só para a prevenção da esquistossomose, mas também de outras doenças que ainda são negligenciadas no país (Barbosa \& Silva, 2018).

Além disso, o estudo desenvolvido por Paz et al. (2021), realizado no Brasil, estados de Alagoas, Pernambuco e Sergipe, observaram que a maior frequência de mortalidade por esquistossomose mansoni estava associada a indivíduos do sexo masculino, idade superior ou igual a 60 anos, cor de pele parda e baixo nível de escolaridade. Assim, esse estudo reforça a necessidade de adequar as intervenções direcionadas as populações vulneráveis, que apresentam maiores probabilidades de serem acometidos pela enfermidade e que possuem déficits na assistência em saúde.

\section{Considerações Finais}

Com base no estudo realizado, conclui-se que o Brasil, apesar dos últimos anos, apresentar uma tendência decrescente nos casos de esquistossomose, ainda constitui um dos países que deve seguir firme no caminho para a atenuação dos impactos da doença. Dessa forma, torna-se indispensável a atuação dos governos federais, estaduais e municipais, em prol de proporcionar as condições básicas de saúde, como água tratada e saneamento básico, para populações desprovidas de tais direitos. Outrossim, é relevante a intervenção das políticas públicas de saúde, no que tange as práticas de educação em saúde, pela qualificação dos profissionais das unidades básicas, que irão transmitir as formas de prevenção, bem como outras orientações sobre a doença, disseminando e popularizando o conhecimento da parasitose na sociedade. Portanto, a pesquisa direcionada ao controle epidemiológico da esquistossomose, torna-se um agente imprescindível, para evidenciar, por meio dos dados e progressões, as regiões que necessitam de intervenções e maior suporte governamental para erradicação desse problema de saúde pública. Dessa forma, é necessária a elaboração de estratégias universais direcionadas à prevenção e controle da esquistossomose, porém enfatizadas sobretudo nas situações de maior vulnerabilidade, visando a diminuição da incidência e prevalência da doença nos grupos mais acometidos e melhorando na qualidade de vida da sociedade.

\section{Referências}

Araújo, M. P., Santos, C. M. A., Gomes, D. S., Cirilo, T. M., Bezerra, L. P., Lima, P. D. \& Santos, I. G. A. (2019). Epidemiologia da esquistossomose em uma área de baixa prevalência de Alagoas entre 2010 e 2016. PUBVET, 14, 139.

Barbosa, L. G. C., \& Silva, J. P. (2019). Esquistossomose e determinantes sociais. Revista Atenas Higeia, 1(2), 41-45.

Brasil. Ministérios de Saúde. Secretaria de Vigilância Epidemiológica. (2021). Boletim epidemiológico: Doenças tropicais negligenciadas. https://www.gov.br/saude/ptbr/media/pdf/2021/marco/3/boletim_especial_doencas_negligenciadas.pdf. 
Carvalho, R. R. S., \& Siqueira, J. H. (2019). Caracterização epidemiológica da esquistossomose no estado do Espírito Santo de 2010 a 2015. Revista Brasileira de Pesquisa em Saúde/Brazilian Journal of Health Research, 21(1), 95-103.

Costa, J. V. B., \& Silva Filho, J. M. (2021). Esquistossomose mansônica: uma análise do perfil epidemiológico na região sudeste. Revista Saúde. com, 17(3).

Gomes, A. C. L., Galindo, J. M., Lima, N. N. D. \& Silva, É. V. G. D. (2016). Prevalência e carga parasitária da esquistossomose mansônica antes e depois do tratamento coletivo em Jaboatão dos Guararapes, Pernambuco. Epidemiologia e Serviços de Saúde, 25, 243-250.

Junior, C. A. R., Dias, F. C. F., Rosa, R. T. A. S., Cardoso, C. R. L., Veloso, F. P. F. S., Mariano, S. M. B., \& Figueiredo, B. N. S. (2017). Esquistossomose na região norte do Brasil. Revista de Patologia do Tocantins, 4(2), 58-61.

Levison, W. L. (2016). Microbiologia e Imunologia Médica. (13a ed.), Artmed, 449-453.

LoVerde, P. T. (2019). Esquistossomose. Advances in experimental medicine and biology, 1154, 45-70. https://doi.org/10.1007/978-3-030-18616-6_3.

Mcmanus, D. P., Dunne D. W., Sacko M., Utzinger J., Vennervald B. J., \& Zhou X. N. (2018). Schistosomiasis. Nature reviews Disease primers, 4(13), 1-19.

Melo, A. G. S. D., Melo, J. J. D. M., Jeraldo, V. D. L. S., \& Melo C. M. (2018). Esquistossomose mansônica em famílias de trabalhadores da pesca de área endêmica de Alagoasa. Escola Anna Nery, 23(1), 1-10.

Nelwan M. L. (2019). Esquistossomose: ciclo de vida, diagnóstico e controle. Current Therapeutic Research, 91(1), 5-9.

Palasio R. G. S., Guimarães, M. C. A., Ohlweiler, F. P., \& Tuan, R. (2017). Identificação molecular e morfológica de espécies de Biomphalaria do estado de São Paulo, Brasil. ZooKeys, (688), 11-32.

Paz, W. S., Duthie, M. S., Jesus, A. R., Araújo, K. C. G. M., Santos, A. D., \& Bezerra-Santos, M. (2021). Modelagem espaço-temporal de base populacional de fatores de risco social e mortalidade por esquistossomose no Brasil entre 1999 e 2018. Acta Tropica, 218, 105897.

Rocha, T. J. M., Santos, M. C. S., Lima, M. V. M., Calheiros, C. M. L., \& Wanderley, F. S. (2016). Aspectos epidemiológicos e distribuição dos casos de infecção pelo Schistosoma mansoni em municípios do Estado de Alagoas, Brasil. Revista Pan-Amazônica de Saúde, 7(2), 6-6.

Santos, A. D., Santos, M. B., Santos, P. G. R., Barreto, A. S., \& Araújo, K. C. G. M. (2016). Análise espacial e características epidemiológicas dos casos de esquistossomose mansônica no município de Simão Dias, nordeste do Brasil. Revista de Patologia Tropical/Journal of Tropical Pathology, 45 (1), 99-114.

Santos, L. S. T. A., \& Cardoso, A. C. C. (2020). Internações por esquistossomose mansônica no estado da Bahia entre 2012 e 2016. Revista Enfermagem Contemporânea, 9(2), 231-237.

Silva, J. P. (2020). Perfil epidemiológico da esquistossomose mansônica em Minas Gerais. Inova Saúde, 9(2), 225-235.

Silva, R. D. M. A., Neto, A. M. A., \& da Silva, E. P. (2021). O uso do praziquantel no tratamento da esquistossomose no município de Limoeiro-PE. Revista Multidisciplinar em Saúde, 2(1), 98-98.

Soares, D. A., Souza, S. A., Silva, D. J., Silva, A. B., Cavalcante, U. M. B., \& Lima, C. M. B. L. (2019). Avaliação epidemiológica da esquistossomose no estado de Pernambuco através de um modelo de regressão beta. Arquivos de Ciências da Saúde, 26(2), 116-120.

Sobrinho, F. S. L., Silva, M. C. S., Lima, L. L. C., Sobrinho, G. K. L., Lopes, E. A. P., \& Feitosa, A. P. S. (2020). Incidência de Esquistossomose Mansônica no Nordeste brasileiro, no período de 2013 a 2017. Diversitas Journal, 5(4), 2881-2889.

Souza, R. L. M., Gargioni, C., Siqueira, R. V., da Silva, R. M., Pinto, P. L. S., \& Kanamura, H. Y. (2017). Aspectos epidemiológicos da esquistossomose em área do sudoeste de Minas Gerais, Brasil. Revista do Instituto Adolfo Lutz, 76, 1-10.

Verjee, M. A. (2019). Esquistossomose: ainda uma causa de morbimortalidade significativa. Pesquisa e relatórios em medicina tropical, $10(1), 153$. 